\title{
Evaluation of Chitosan Based Polymeric Matrices for Sustained Stomach Specific Delivery of Propranolol Hydrochloride
}

\author{
Juhi Dubey, Anurag Verma, and Navneet Verma \\ Department of Pharmaceutics, IFTM University, Moradabad 244001, India \\ Correspondence should be addressed to Anurag Verma; anuragverma_iftm@yahoo.co.in
}

Received 29 May 2015; Revised 9 July 2015; Accepted 14 July 2015

Academic Editor: Marino Lavorgna

Copyright ( 2015 Juhi Dubey et al. This is an open access article distributed under the Creative Commons Attribution License, which permits unrestricted use, distribution, and reproduction in any medium, provided the original work is properly cited.

\begin{abstract}
The objective of the present investigation was to explore the potential of Chitosan based polymeric matrices as carrier for sustained stomach specific delivery of model drug Propranolol Hydrochloride. Briefly, single unit hydrodynamically balanced (HBS) capsule formulations were prepared by encapsulating in hard gelatin capsules, intimately mixed physical mixtures of drug, and cationic low molecular weight Chitosan (LMCH) in combination with either anionic medium viscosity sodium alginate (MSA) or sodium carboxymethylcellulose $(\mathrm{CMCNa})$. The effect of incorporation of nonionic polymers, namely, tamarind seed gum (TSG) and microcrystalline cellulose (MCCP), was also investigated. It was observed that HBS formulations remained buoyant for up to $6 \mathrm{~h}$ in $0.1 \mathrm{M} \mathrm{HCl}$, when $\mathrm{LMCH}$ : anionic/nonionic polymer ratio was at least $4: 1$. It was also observed that $\mathrm{LMCH}$ has formed polyelectrolyte complex (PEC) with MSA ( $4: 1.5$ ratio) and CMCNa (4:1 ratio) in situ during the gelation of HBS formulations in $0.1 \mathrm{M} \mathrm{HCl}$. The retardation in drug release was attributed to the PEC formation between LMCH and MSA/CMCNa. Incorporation of MCCP (rapid gel formation) and TSG (Plug formation) was found to be innovative. From the data, it is suggested that Chitosan based polymeric matrices may constitute an excellent carrier for stomach specific drug delivery.
\end{abstract}

\section{Introduction}

It has always been difficult to engineer polymers or polymeric compositions for stomach specific drug delivery [1-3]. The engineered polymeric compositions should have attributes that are pertinent to high level of gastric retention, generally $5-6 \mathrm{~h}[4,5]$, release the drug at zero-order or at a constant rate $[6,7]$, and degrade in vivo to smaller fragments, which can then be excreted from the body; their degradation products must be nontoxic and not create an inflammatory response; and finally, they should degrade within a reasonable period of time $[1,2]$. Although polymers of various origins are available and used in drug delivery systems, natural polysaccharides find much application because of their favorable characteristics like abundant availability, inexpensive, nontoxic, noncarcinogenic, and biodegradable, and more importantly biocompatibility. Of these, Chitosan has received a great deal of attention due to its excellent biocompatibility, biodegradability, and nontoxicity. Chitosan fulfills all the polymeric attributes that are pertinent to high level of retention at applied and targeted sites via mucoadhesive bonds. The mucoadhesive property of Chitosan is due to electrostatic interaction of the protonated amino group in Chitosan with negatively charged silicic acid residues in mucin (the glycoprotein that composes the mucus). This interaction takes place very close to the mucosal surface and thus possesses potential to confer significant gastroretention to the hydrogel. Additionally, the hydroxyl and amino groups may interact with mucus via hydrogen bonding. The linearity of Chitosan molecules also ensures sufficient flexibility for interpenetration. Further, it also possesses cell-binding activity due to its polymer cationic polyelectrolyte structure and to the negative charge of the cell surface [8-11]. Although this material has already been extensively investigated in the design of different types of drug delivery systems, it is still less explored for stomach specific drug delivery systems. The objective of the present investigation is to explore the potential of this wonderful material in combination with anionic/nonionic natural polysaccharides like sodium alginate, sodium carboxymethylcellulose, microcrystalline cellulose, and tamarind seed gum in the fabrication of stomach specific single unit HBS capsule formulations using 
TAble 1: Composition of HBS capsules containing Propranolol HCl.

\begin{tabular}{|c|c|c|c|c|c|c|}
\hline Formulation code & LMCH (mg) & MSA (mg) & $\mathrm{MCCP}(\mathrm{mg})$ & $\mathrm{CMCNa}(\mathrm{mg})$ & TSG (mg) & PHCL (mg) \\
\hline $\mathrm{J} 1$ & 200 & 50 & & & & 50 \\
\hline $\mathrm{J} 2$ & 200 & 75 & & & & 50 \\
\hline $\mathrm{J} 3$ & 200 & 50 & 10 & & & 50 \\
\hline $\mathrm{J} 4$ & 200 & & & 50 & & 50 \\
\hline J5 & 200 & & & 40 & 10 & 50 \\
\hline J6 & 200 & 40 & & & 10 & 50 \\
\hline J7 & 200 & 40 & 10 & & & 50 \\
\hline J8 & 50 & 200 & & & & 50 \\
\hline J9 & & 200 & & & & 50 \\
\hline
\end{tabular}

Propranolol Hydrochloride as model drug. The proposed combinations of cationic (Chitosan) and anionic polymers (sodium alginate/carboxymethylcellulose) are expected to form a low density hydrogel that remained buoyant on acidic dissolution medium $(0.1 \mathrm{M} \mathrm{HCl})$ and sustain the release of highly hydrophilic model drug by in situ polyelectrolyte complex formation.

\section{Material and Methods}

2.1. Materials. Propranolol Hydrochloride (PHCL) was obtained as a gift sample from Dr. Reddy's Laboratories, India. Low molecular mass Chitosan (LMCH) [viscosity $20.00 \mathrm{cps}$ of $1 \%$ in $1 \%$ acetic acid and degree of deacetylation (DD) $>80 \%$, medium viscosity sodium alginate (MSA) $\left[\geq 2,000 \mathrm{cps}, 2 \%\right.$ in water at $25^{\circ} \mathrm{C}$, and carboxymethylcellulose sodium [CMCNa, average $\mathrm{Mw} \sim 90,000]$ were procured from Sigma Aldrich Brookfield. Tamarind seed gum (TSG) and microcrystalline cellulose (MCCP) [PH 102] were procured from the Central Drug House, India. All other chemicals used were of analytical grade.

\subsection{Methods}

2.2.1. Preparation of HBS Capsules Containing Propranolol $\mathrm{HCl}$ (PHCL). Single unit capsules were prepared by physically blending PHCL with the required quantity of polymers as mentioned in Table 1, using double cone blender for $15 \mathrm{~min}$, followed by encapsulation into hard gelatin capsules [12].

\subsubsection{In Vitro Evaluation of HBS Capsule Formulations}

(1) In Vitro Buoyancy Studies [12, 13]. Prepared HBS capsules were immersed in $0.1 \mathrm{M}$ hydrochloric acid $\left(\mathrm{pH} 1.2,37 \pm 0.5^{\circ} \mathrm{C}\right)$ in USP paddle type apparatus at $50 \mathrm{rpm}$. The time for which the capsules remained buoyant was observed.

(2) Determination of Drug Contents of HBS Capsules [12, 13]. PHCL contents were determined by emptying $10 \mathrm{HBS}$ capsules from each formulation as completely as possible. A powder equivalent to average weight was added to $100 \mathrm{~mL}$ $0.1 \mathrm{M} \mathrm{HCl}\left(\mathrm{pH} 1.2,37 \pm 0.5^{\circ} \mathrm{C}\right)$, followed by stirring for one hour at $500 \mathrm{rpm}$. The solution was filtered through a $0.45 \mu$ membrane filter and diluted suitably and the absorbance of resultant solution was measured spectrophotometrically at $290 \mathrm{~nm}$.

(3) In Vitro Drug Release Studies. In vitro release of PHCL from the HBS capsule formulations was performed in USP dissolution apparatus type II at $50 \mathrm{rpm}$. Evaluation of drug release was performed by using $900 \mathrm{~mL} 0.1 \mathrm{M} \mathrm{HCl}(\mathrm{pH} 1.2)$ at $37 \pm 0.5^{\circ} \mathrm{C}$. At predetermined intervals, one $\mathrm{mL}$ aliquot was withdrawn and replenished with an equal volume of fresh dissolution medium. Withdrawn samples were analyzed spectrophotometrically at $290 \mathrm{~nm}$.

2.2.3. Drug Release Mechanism. Different kinetic models such as zero-order, first-order, and square root (Higuchi) can be applied to interpretation of drug release kinetics. A zeroorder release refers to a uniform or nearly uniform rate of release of a drug from the solid dosage form after coming in contact with an aqueous environment, independent of the drug concentration in the dosage form during a given time period. Dosage forms with zero-order release generally provide maximum therapeutic value with minimal side effects. For many extended release formulations, the rate of drug release initially increases rapidly followed by decreased rate of drug release. This type of drug release is categorized as the first-order release. Such dosage form may not produce uniform concentration levels of the drug in the systemic circulation for a prolonged period of time. The Higuchi release equation predicts that the drug release is caused primarily by diffusion mechanism [14]:

$$
Q=K \sqrt{t}
$$

where $Q$ is the amount of the drug released in time $t$ and $K$ is the release constant from the equation. The data were also subjected to Korsmeyer-Peppas power law [15] as in (2). The Korsmeyer-Peppas model provides an insight into the type of drug release mechanism taking place from swellable polymeric matrices:

$$
\frac{M t}{M \infty}=K t^{n}
$$

where $M_{t} / M_{\infty}$ is the fraction of drug released in time $t, K$ is the structural and geometric constant, and $n$, the release exponent, is estimated from linear regression fit of the 
logarithmic release data. Practically, one has to use the first $60 \%$ of a release curve to determine the slope obtained from (2) regardless of the geometric shape of the delivery device. A good fit to the Korsmeyer-Peppas equation indicates the combined effect of diffusion and relaxation mechanisms for the release.

2.2.4. Statistical Analysis. The differences in average data were compared by simple analysis of variance (one-way analysis of variance) or Student's $t$-test (SigmaPlot 11).

\section{Result and Discussion}

Hydrodynamically balanced (HBS) capsule formulations are the simplest gastroretentive dosage forms. These systems are usually composed of hard gelatin capsules filled with a mixture of gel-forming polymeric substances and an active pharmaceutical ingredient. After immersion in simulated stomach fluid/acidic dissolution medium ( $\mathrm{pH}$ 1.2) (in vitro) or swallowing (in vivo), the shell of the swollen hydrogel is formed. It controls the release rate of the drug, and it maintains appropriate integrity of the HBS and low apparent density of the systems, ensuring flotation. Such systems are best suited for drugs having a better solubility in acidic environment and for the drugs having specific site of absorption in the upper part of the small intestine [16].

3.1. In Vitro Buoyancy Studies. In vitro buoyancy studies were carried out in $0.1 \mathrm{M} \mathrm{HCl}(\mathrm{pH} 1.2)$ maintained at $37 \pm 0.5^{\circ} \mathrm{C}$ using USP dissolution apparatus type II at $50 \mathrm{rpm}$.

For efficient buoyancy, swelling of polymer is very vital. LMCH contains $-\mathrm{NH}_{2}$ - groups bound to polymer chains. In the presence of acidic gelation medium, the polymer chains in $\mathrm{LMCH}$ absorb dissolution medium and the binding of $\mathrm{H}^{+}$ causes the polymer to swell $\left(\mathrm{NH}_{3}{ }^{+}\right)$. The air entrapped in the swollen polymeric network is expected to maintain the density less than unity which ultimately confers buoyancy to the hydrogel for extended period of time [17]. It was observed that the HBS capsule formulations prepared with PHCL and LMCH alone sank within h. The poor buoyancy could be attributed to the weak gel network formed due to the presence of highly soluble PHCL $(225 \mathrm{mg} / \mathrm{mL}$ in $0.1 \mathrm{M}$ $\mathrm{HCl}$ at $20^{\circ} \mathrm{C}$ ) in $\mathrm{LMCH}$ based formulation that could not hold drug particles in the gel network. Therefore, to prolong the buoyancy, the auxiliary polymers (MSA, CMCNa, and TSG) were incorporated into HBS capsule formulations. These auxiliary polymers are expected to counter the rapid erosion of gel layer, thereby maintaining the integrity of the swollen hydrogel. All the formulations exhibited immediate buoyancy with no lag time (Table 2). HBS capsule formulations, J1J6, remained buoyant up to $6 \mathrm{~h}$, whereas formulations J7-J9 remained buoyant for $5 \mathrm{~h}$ (J7) and $4 \mathrm{~h}$ (J8 and J9), respectively.

It was also observed that $\mathrm{LMCH}$ : auxiliary polymer ratio is critical for buoyancy. The formulations remained buoyant for prolonged period when the $\mathrm{LMCH}$ : auxiliary polymer ratio was $4: 1$ (LMCH : MSA; LMCH : CMCNa; LMCH : MSA + TSG; LMCH : CMCNa + TSG). The addition of auxiliary polymer(s) resulted in improvement in swelling of the $\mathrm{LMCH}$
TABLE 2: In vitro floating characteristics of HBS formulations.

\begin{tabular}{lcc}
\hline Formulation code & $\begin{array}{c}\text { Floating lag time } \\
\text { (Seconds) }\end{array}$ & $\begin{array}{c}\text { Duration of } \\
\text { floating }(\mathrm{hrs})\end{array}$ \\
\hline J1 & Nil & Up to $6 \mathrm{hrs}$ \\
J2 & Nil & Up to $6 \mathrm{hrs}$ \\
J3 & Nil & Up to $6 \mathrm{hrs}$ \\
J4 & Nil & Up to $6 \mathrm{hrs}$ \\
J5 & Nil & Up to $6 \mathrm{hrs}$ \\
J6 & Nil & Up to $6 \mathrm{hrs}$ \\
J7 & Nil & Sank after $5 \mathrm{hrs}$ \\
J8 & Nil & Sank after $4 \mathrm{hrs}$ \\
J9 & Nil & Sank after $4 \mathrm{hrs}$ \\
\hline
\end{tabular}

based formulations which in turn resulted in increase in bulk volume. The purpose of incorporating insoluble MCCP into the HBS formulation was to increase the porosity of the polymer matrix in order to improve the hydration and subsequent gel formation. It was observed that the whole polymeric matrix was hydrated within $15 \mathrm{~min}$ (J3 and J7) compared to $60 \mathrm{~min}$ in case of formulation J1. This fast hydration has resulted in rapid gelling but there was no improvement in buoyancy (J3) and in case of formulation J7, buoyancy was even decreased to $5 \mathrm{~h}$. This could be attributed to the formation of a swollen hydrogel, whose density is greater than unity, due to excessive imbibition of aqueous acidic medium. Reversing the $\mathrm{LMCH}$ : MSA ratio $(1: 4, \mathrm{~J} 8)$ or completely removing LMCH with MSA (J9) resulted in decreased in vitro buoyancy. The observed behavior (J8 and J9) could be attributed to the poor swelling of MSA in acidic dissolution medium coupled with the high diffusional driving force exerted by highly hydrophilic PHCL, which resulted in rapid erosion of gel layer leading to loss of gel integrity.

\subsection{Determination of Drug Contents of HBS Capsule Formula-} tions. The drug content determination test is done to ensure that each HBS capsule formulation contains equal amount of drug. For this purpose encapsulated contents of $10 \mathrm{HBS}$ capsules from each formulation were emptied as completely as possible. The contents so removed were then put into $100 \mathrm{~mL} 0.1 \mathrm{M} \mathrm{HCl}\left(\mathrm{pH} 1.2,37 \pm 0.5^{\circ} \mathrm{C}\right)$ and stirred for one hour at $500 \mathrm{rpm}$. The solution was filtered through a $0.45 \mu$ membrane filter and diluted suitably and the absorbance of resultant solution was measured spectrophotometrically at $290 \mathrm{~nm}$. Drug contents of various formulations are given in Table 3. All capsule formulations were found to contain PHCL contents within limit [13].

3.3. In Vitro Drug Release Studies. In the present study, we have chosen Chitosan because of its ability to release loaded drugs slowly in the stomach, since the gel formation by cationic Chitosan is pronounced at acidic $\mathrm{pH}$, which results in marked retardant effects on drug release $[9,12]$. During the preliminary formulation development studies, we have investigated the feasibility of LMCH alone as a carrier for stomach specific delivery of highly hydrophilic model drug. 
TABLE 3: PHCL contents in various HBS formulations.

\begin{tabular}{lc}
\hline Formulation code & Percentage (\%) of PHCL contents \\
\hline J1 & $99.49 \pm 2.98$ \\
J2 & $99.91 \pm 2.93$ \\
J3 & $99.65 \pm 3.12$ \\
J4 & $99.75 \pm 2.11$ \\
J5 & $99.89 \pm 1.91$ \\
J6 & $101.4 \pm 3.78$ \\
J7 & $99.67 \pm 2.58$ \\
J8 & $99.96 \pm 2.49$ \\
J9 & $99.79 \pm 2.39$ \\
\hline
\end{tabular}

However, polymeric matrices composed of LMCH (200 mg) and PHCL $(50 \mathrm{mg})$ could not float for more than one hour and released almost $80 \%$ of PHCL (Burst release) before sinking in the acidic dissolution medium. This could be explained as, being cationic in nature, swelling of Chitosan in acidic gelation medium will be a more entropy-favored process and as the number of ions within the hydrogel structure increases, more and more osmotic and electrostatic forces will be created within the hydrogel structure [18]. This leads to increased dissolution medium $(0.1 \mathrm{M} \mathrm{HCl})$ uptake and forces a typical hydrogel to behave thermodynamically like a liquid as it occupies more space. Moreover, Propranolol is highly hydrophilic, which further reduces the strength of the aqueous gel layer due to high diffusional driving force and consequently increased erosion. As a result Chitosan hydrogel lost its integrity and became distorted leading to burst release.

Considering the above observation, in the present study, to improve the buoyancy and to address the problem of burst release, we have decided to combine LMCH with MSA or CMCNa. It is expected that such a polymeric composition not only maintains the integrity of the hydrogel and thus buoyancy, but also is expected to form in situ polyelectrolyte complex (PEC) between LMCH and MSA or CMCNa. This PEC formation is expected to retard the dilution of outer gel layer of swellable and erodible hydrogel, thereby, retarding the diffusion of PHCL. It has been reported that the PECs formed between a polycation (e.g., LMCH) and polyanion (e.g., MSA or CMCNa) exhibit a very high degree of ordering and crystal like properties and have quite compact structures and are little affected by $\mathrm{pH}$ variation of the dissolution medium [19]. Keeping this in view, it is expected that if these complexes are formed in situ it might be possible to overcome the initial burst release of PHCL.

From the formulation J1, about $65.2 \%$ of PHCL (Figure 1) was released within first $h$, after that remaining drug was released in a slower manner. This observation is opposite to our expectation, that is, retarded drug release. This could be explained as there must be sufficient polymer contents in a matrix to form a uniform barrier. This barrier protects the drug from immediately releasing into the dissolution medium. In this case, it is possible that a uniform gel layer might not have formed to retard the PHCL release.

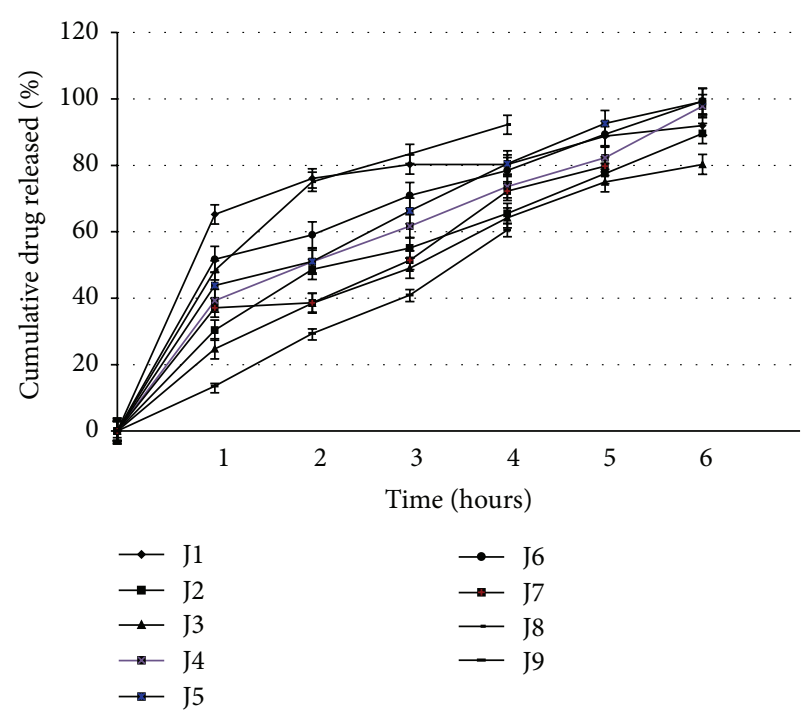

FIGURE 1: Cumulative drug release (\%) of formulations J1-J9.

Another reason could be the delayed gel formation. It was observed that when the formulation J1 was placed in dissolution media, due to imbibition of the acidic dissolution medium, the hard gelatin capsule shell disrupted $(15 \mathrm{~min})$ and a gel layer around the polymer matrix were formed ( $\sim 30 \mathrm{~min})$. It seems that during the first $30 \mathrm{~min}$ most of the PHCL particles located at the surface of the polymer matrix, dissolved and released rapidly.

Therefore, in formulation J2, it was decided to study the effect of increased concentration of MSA (LMCH : MSA ratio $4: 1.5)$ in polymer matrix keeping the $\mathrm{LMCH}$ concentration constant. In this case significant retardation $(p<0.01$, J1 and J2) in drug release which was observed with only $30 \%$ of PHCL (Figure 1) was released within first hour. This could be attributed to quick formation of a hydrogel in which polyelectrolyte complex (PEC) might have formed in situ. The relatively quick gel formation could be attributed to the association/dissociation/binding of various ions to the polymer chains within the hydrogel structure exposed to acidic dissolution medium [20].

To confirm PEC formation (J2) between oppositely charged polymers, Differential Scanning Calorimetric (DSC) on dried gelled polymeric compositions was carried out. Briefly formulation composition which corresponded to blank J2 was accurately weighed and put into bags made up of dialysis membrane (1000-molecular weight cutoff, Sigma Aldrich). The bags were then heat sealed on both sides and exposed to the acidic dissolution medium using USP type II dissolution apparatus. After $30 \mathrm{~min}$ of exposure to dissolution medium $(0.1 \mathrm{M} \mathrm{HCl}, \mathrm{pH} 1.2)$, the contents of the bags were removed and dried in an oven overnight at $60^{\circ} \mathrm{C}$ and DSC Thermogram (TA Instruments, USA, Model: SDT 2960) was recorded $\left(50\right.$ to $400^{\circ} \mathrm{C}$ at a heating rate of $10^{\circ} \mathrm{C} / \mathrm{min}$ ). Nitrogen was employed as blanket gas. The characteristic peaks (endotherm and exotherm) were recorded. The DSC Thermograms of LMCH and MSA were 


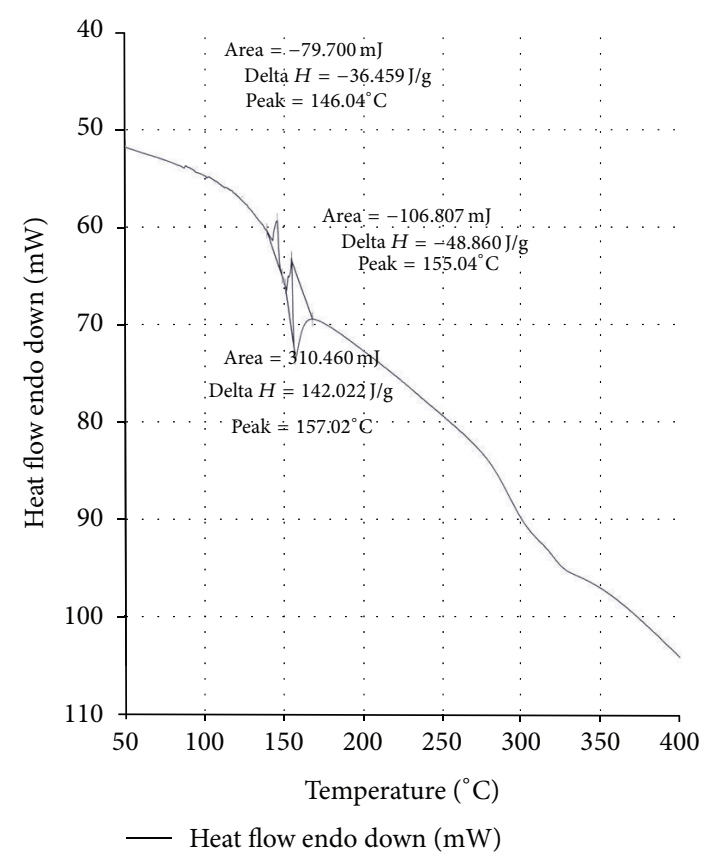

(a)

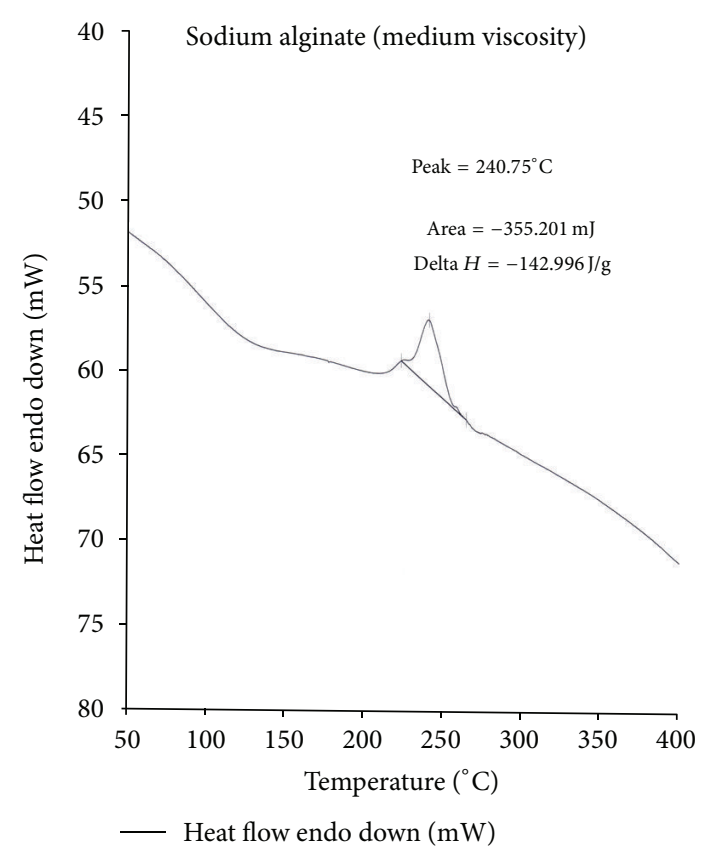

(b)

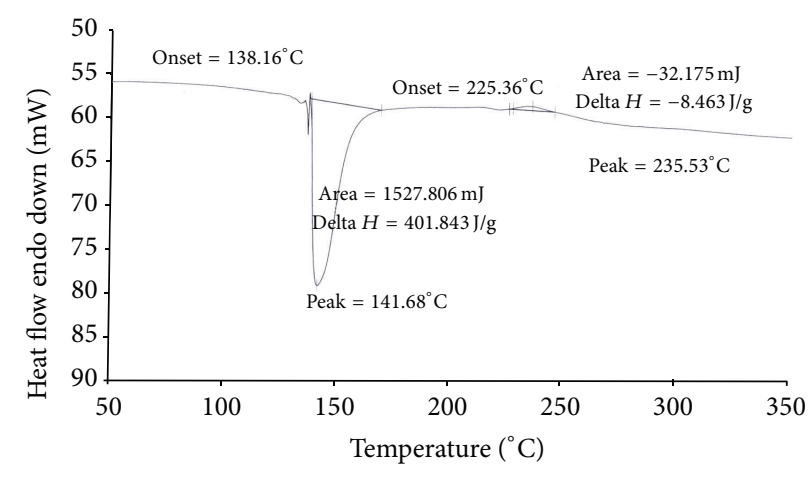

- Heat flow endo down $(\mathrm{mW})$

(c)

Figure 2: (a) DSC Thermogram of LMCH. (b) DSC Thermogram of MSA. (c) DSC Thermogram of LMCH + MSA dried gel.

also recorded under identical conditions for comparison and confirmation purposes.

The DSC Thermogram of LMCH (Figure 2(a)) showed a broad endothermic peak at $157^{\circ} \mathrm{C}$, which indicates the glass transition temperature of polymer.

The DSC Thermogram (Figure 2(b)) of pure MSA showed a broad exothermic peak at $240^{\circ} \mathrm{C}$ indicating slow degradation of MSA.

The DSC Thermogram obtained from dried gel sample (blank J2) revealed an endothermic peak at $141^{\circ} \mathrm{C}$ and an exothermic peak $235^{\circ} \mathrm{C}$ (Figure 2(c)). Both these peaks were missing in the individual thermograms of polymers ( $\mathrm{LMCH}$ and MSA). The first broad endothermic peak at $141^{\circ} \mathrm{C}$ could be attributed to the glass transition temperature of $\mathrm{LMCH}$ MSA polyelectrolyte complex. The second exothermic peak at $235^{\circ} \mathrm{C}$ is very weak and could be attributed to the slow degradation of polyelectrolyte complex. The formed LMCHMSA polyelectrolyte complex exhibited its capability of modulating the initial burst as well as the subsequent sustained release of the PHCL.

The PEC formation between LMCH and MSA was also confirmed by Fourier Transform Infrared (FTIR) Spectroscopy. To deduce PEC formation, the FTIR (Shimadzu, model 8400S) spectra of LMCH, MSA, and dried gel sample were recorded and compared. The samples were prepared in $\mathrm{KBr}$ disks (2 $\mathrm{mg}$ sample in $200 \mathrm{mg} \mathrm{KBr}$ ). The scanning range was $400-4000 \mathrm{~cm}^{-1}$ and the resolution was $2 \mathrm{~cm}^{-1}$. The FTIR spectra (Figure 3(a)) of MSA showed major absorption bands at 1610 and $1402 \mathrm{~cm}^{-1}$ due to asymmetric and symmetric stretching bond of carboxyl group. The IR absorption band at $3434 \mathrm{~cm}^{-1}$ is attributed to $\mathrm{O}-\mathrm{H}$ stretching. 


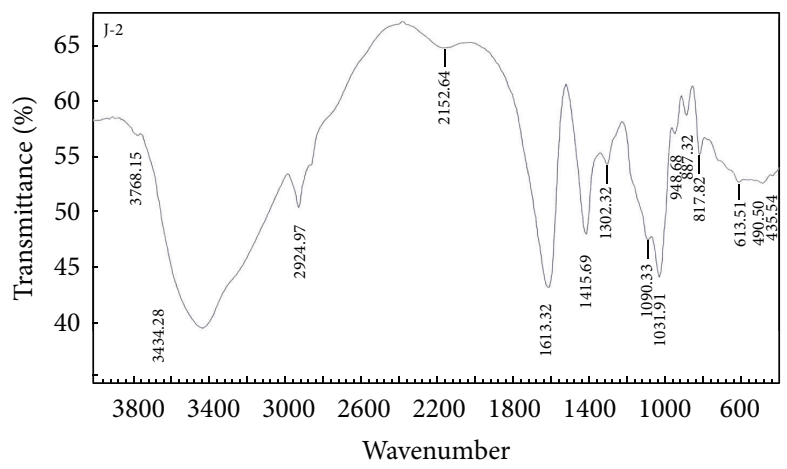

(a)

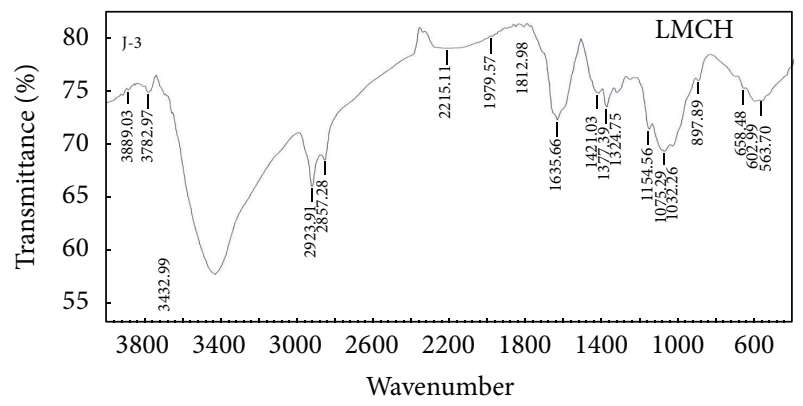

(b)

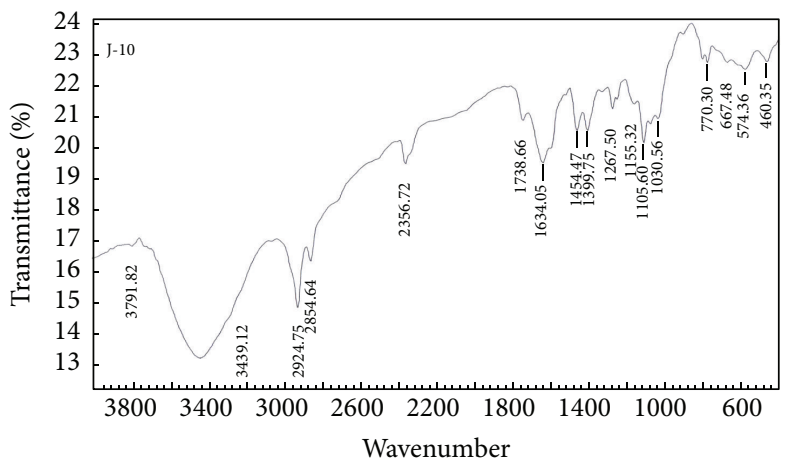

(c)

FIGURE 3: (a) FTIR spectra of MSA. (b) FTIR spectra of LMCH. (c) FTIR spectra of LMCH + MSA dried gel.

The FTIR spectra of LMCH (Figure 3(b)) exhibited absorption band at 1635,1421 , and $1377 \mathrm{~cm}^{-1}$ due to amides I, II, and III. Absorption band at 3412.3 and $2923.9 \mathrm{~cm}^{-1}$ is due to hydroxyl stretch and $\mathrm{C}-\mathrm{H}$ stretch.

The FTIR spectra of dried gel sample (Figure 3(c)) exhibited shifting in absorption bands together with appearance of a new IR absorption band. The absorption band due to amides I, II, and III in LMCH was shifted to 1654 , 1454 , and $1309 \mathrm{~cm}^{-1}$, indicating change in the environment and there was a new band at $1730 \mathrm{~cm}^{-1}$. Further, the FTIR spectrum also showed shifting of $\mathrm{O}-\mathrm{H}$ stretching vibration to $3444.40 \mathrm{~cm}^{-1}$ from $3418 \mathrm{~cm}^{-1}$ attributed to the free $-\mathrm{OH}$ groups of both polymers existing in the PEC structure. All these observations could be suggestive of the PEC formation between LMCH and MSA.

From formulation J3, only $24.74 \%$ of PHCL (Figure 1) was released within first hour. Now this is interesting, as we have already showed that, below the $75 \mathrm{mg}$ MSA concentration, PEC formation with LMCH was not observed and then how could the PHCL release was retarded significantly $(p<0.042$, $\mathrm{J} 1$ and J3). The formulation J3 was composed of $\mathrm{LMCH}, \mathrm{MSA}$, and MCCP $(200+40+10 \mathrm{mg})$. The purpose of incorporating MCCP was to increase the porosity of the polymer matrix in order to improve the hydration of polymer matrix. It was observed that the whole polymeric matrix was hydrated within $15 \mathrm{~min}$ compared to $60 \mathrm{~min}$ in case of formulation $\mathrm{J1}$.
This fast hydration has resulted in rapid gelling and thus slow diffusion of PHCL.

In case of formulation $\mathrm{J} 4$, an attempt has been made to study the release of PHCL from LMCH-CMCNa PEC gel. For this purpose MSA was replaced with $\mathrm{CMCNa}$ in equal amount, that is, $50 \mathrm{mg}$. The PHCL release (Figure 1) was found to be significantly retarded compared $(39.24 \%$ at the end of first h) to formulation J1 ( $p<0.00477$, J1 and J4). Here also, in order confirm in situ PEC formation, the DSC studies on dried gelled samples [LMCH $+\mathrm{CMCNa}(4: 1$, J4 blank) prepared in the same manner as $\mathrm{LMCH}+\mathrm{MSA}$ dried gel] and individual polymers were carried out.

The DSC Thermogram of pure CMCNa (Figure 4(a)) showed an endothermic peak at $144.33^{\circ} \mathrm{C}$ corresponding to the glass transition temperature of the polymer.

The DSC Thermogram of $\mathrm{LMCH}+\mathrm{CMCNa}$ dried gel (Figure 4(b)) showed two endothermic peaks at $150^{\circ} \mathrm{C}$ and $219^{\circ} \mathrm{C}$. Both of these peaks are missing in the individual thermogram of $\mathrm{LMCH}$ and $\mathrm{CMCNa}$. The first broad endothermic peak at $150^{\circ} \mathrm{C}$ could be attributed to the glass transition temperature of $\mathrm{LMCH}-\mathrm{CMCNa}$ polyelectrolyte complex. The second exothermic peak at $219^{\circ} \mathrm{C}$ is very weak and could be attributed to the slow degradation of polyelectrolyte complex.

The PEC formation between LMCH and CMCNa was also confirmed by FTIR spectroscopy studies. Here also the FTIR spectra of $\mathrm{LMCH}, \mathrm{CMCNa}$, and $\mathrm{LMCH}+\mathrm{CMCNa}$ dried gel were recorded and compared. The FTIR spectra 


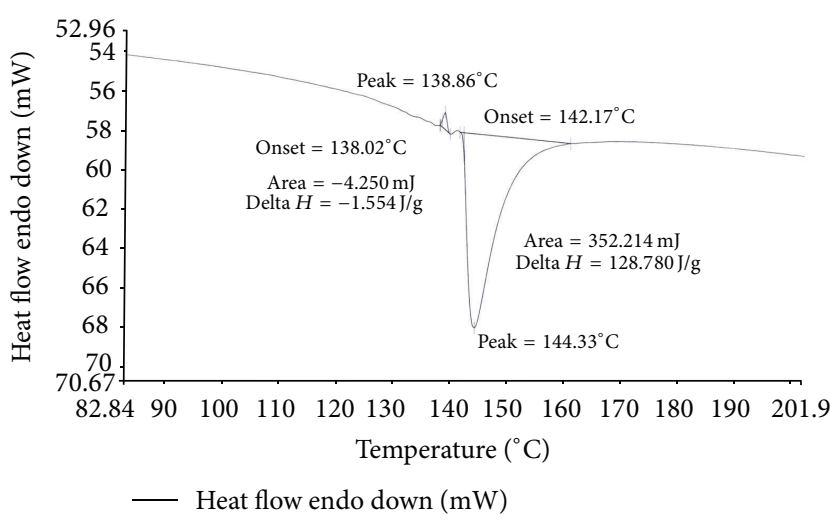

(a)

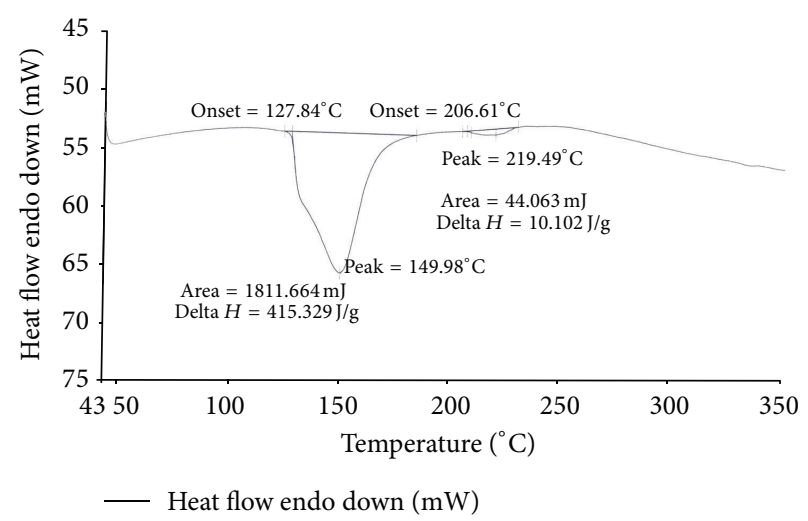

(b)

Figure 4: (a) DSC Thermogram of CMCNa. (b) DSC Thermogram of LMCH + CMCNa dried gel.

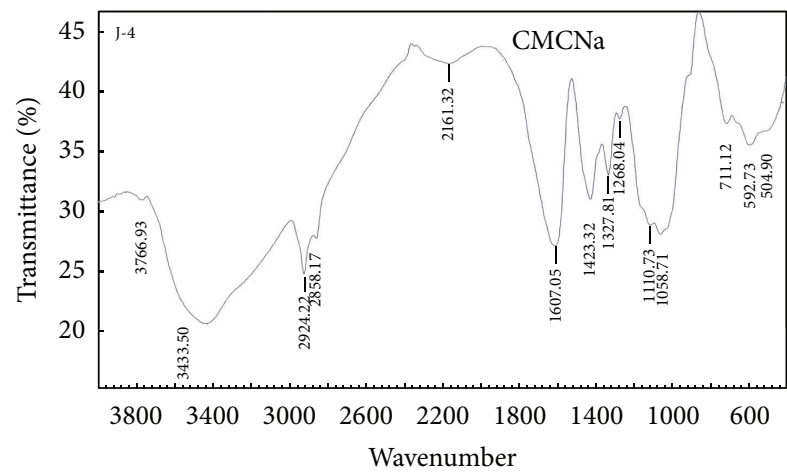

(a)

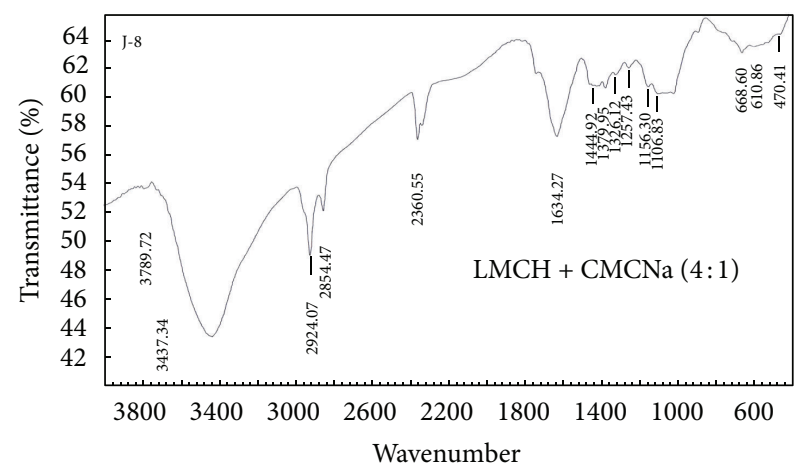

(b)

FIGURE 5: (a) FTIR spectra of CMCNa. (b) FTIR spectra of LMCH + CMCNa dried gel.

of $\mathrm{CMCNa}$ (Figure 5(a)) showed major absorption bands at $1058,1110,1607$, and $1423 \mathrm{~cm}^{-1}$ attributed to stretching vibrations of $\mathrm{C}-\mathrm{OH}$ group, $\mathrm{C}-\mathrm{OH}$ group, and symmetric and asymmetric vibrations of carboxylate group.

The FTIR spectra of $\mathrm{LMCH}+\mathrm{CMCNa}$ dried gel (Figure 5(b)) exhibited shifting of absorption bands. The absorption band due to symmetric and asymmetric vibrations of carboxylate group was shifted to $1654 \mathrm{~cm}^{-1}$ and $1444 \mathrm{~cm}^{-1}$. Although no new peak has appeared in the spectra, there is change in environment of carboxyl group, suggesting PEC formation.

In formulation J5, some amount of MSA was replaced with tamarind seed polysaccharide (TSG). Tamarind seed polysaccharide is a nonionic, neutral, branched polysaccharide comprising a cellulose-like backbone. It is dispersible in warm mater to form a highly viscous gel (up to $2800 \mathrm{cps}$ in $3 \%$ solution) as a mucilaginous solution with a broad $\mathrm{pH}$ tolerance and adhesivity. It possesses properties such as mucomimetic, mucoadhesive, and pseudoplastic properties. It is a multifunctional polymer, which plays the role of release retardant, modifier, and a carrier for novel drug delivery systems for oral, buccal, colon, ocular systems and so forth [21]. It was expected that incorporation of TSG to the LMCHMSA matrix will increase viscosity of gelled polymer matrix which in turn offer more resistance to diffusion of PHCL through the gelled polymer matrix. It was observed that addition of TSG resulted in the formation of a polymer matrix in the form of plug rather than random network of gelled mass. However, contrary to our expectation, about $51 \%$ of PHCL (Figure 1) was released within first h; this could be attributed to time period spent on plug formation. When the HBS formulations (J5) were placed in $0.1 \mathrm{M} \mathrm{HCl}$, dissolution medium begins to penetrate into the polymer matrix through the disrupted capsule shells. Total time spent on plug formation ranged from 18 to $20 \mathrm{~min}$ and during this period most of the highly water soluble drug was leached out of the system. Once the plug formation was complete the PHCL release was significantly retarded with next 50\%; PHCL was released in about $5 \mathrm{~h}(9,11,8,11$, and $9 \%$ at the end of $2,3,4,5$, and 6 th $\mathrm{h}$ ). In formulation $\mathrm{J} 6$, here also the plug formation was observed but the release retardant effect was not statistically different compared to J5 $(p<0.2599$, J5 and J6). In case of formulation J7, effect of incorporation of MCCP was again studied, this time with $\mathrm{LMCH}-\mathrm{CMCNa}$ polymer matrix. As expected polymer hydration took place rapidly and about $37 \%$ of drug was released within first hour (Figure 1). It was also observed that the release profiles compared to formulation J4 were 
TABLE 4: Drug release kinetics of HBS formulations of PHCL.

\begin{tabular}{|c|c|c|c|c|c|}
\hline Formulation code & Zero order & First order & Higuchi & Korsmeyer-Peppas & $n$ value \\
\hline $\mathrm{J1}$ & 0.6399 & 0.9620 & 0.8814 & - & $0.54^{*}$ \\
\hline $\mathrm{J} 2$ & 0.9471 & 0.9193 & 0.9945 & 0.8782 & 0.41 \\
\hline $\mathrm{J} 3$ & 0.9682 & 0.9851 & 0.9772 & 0.8614 & 0.51 \\
\hline $\mathrm{J} 4$ & 0.9314 & 0.7755 & 0.9975 & 0.8742 & 0.41 \\
\hline J5 & 0.9234 & 0.8674 & 0.9844 & 0.8072 & 0.43 \\
\hline J6 & 0.8577 & 0.8106 & 0.9728 & 0.8772 & 0.41 \\
\hline J7 & 0.9253 & 0.9258 & 0.9655 & 0.7018 & 0.24 \\
\hline J8 & 0.8685 & 0.9888 & 09881 & - & 1.28 \\
\hline J9 & 0.9948 & 0.9668 & 0.8933 & 0.8753 & 0.52 \\
\hline
\end{tabular}

${ }^{*}$ Determined for amount of drug released during the first $\mathrm{h}$ at $15 \mathrm{~min}$ interval.

almost similar. This suggests that PEC formation could be the main mechanism of PHCL release retardation rather than fast gelling of polymer matrix. In formulation $\mathrm{J} 8$, the ratio of LMCH:MSA was reversed compared to formulation J1. This has resulted in significantly decreased buoyancy with no statistically different release profile $(p<0.3663$, J1 and J8). The same observation was obtained when LMCH was completely replaced with MSA. The polymer matrix could not float for more than $4 \mathrm{~h}$; however, release profile was significantly $(p<0.013 \mathrm{~J} 8$ and $\mathrm{J} 9)$ retarded.

3.4. Mechanism of Drug Release. From the data generated from kinetic analysis, HBS capsule formulations J1, J3, and J8 followed first-order release pattern as evidenced by their $r^{2}$ values (Table 4 ). In this case, at first there is a burst effect where the drug is rapidly dissolved and released from the surface before the gel membrane is fully formed at the surface. In the second part, the release rate decreases continuously until the end of the process. This release pattern is characteristic of first-order release kinetics. On the other hand, formulations J2 and J4-J7 followed Higuchi release kinetics as evidenced by their $r^{2}$ values. Higuchi model states that the accumulative drug release from a swollen hydrogel is diffusion controlled and is proportional to the square root of the time. This model assumes a pseudosteady state, constant diffusivity, rapid drug dissolution, and sinks conditions. The Higuchi model is applicable if the release is largely governed by diffusion through water-filled pores in the matrix [14].

A good fit to the Higuchi model together with low $n$ values determined by the Korsmeyer-Peppas equation (formulations J2 and J4-J7) suggests that the swelling of Chitosan based polymer matrices and resultant drug release in $0.1 \mathrm{M} \mathrm{HCl}$ is diffusion-controlled. Formulation code $\mathrm{J9}$ followed zero-order release kinetics, a release rate that is substantially constant over time.

The $n$ values of various Chitosan based HBS formulations calculated from Korsmeyer-Peppas equation are given in Table 4. For polymeric slabs, cylinders, and spherical systems, $n=0.5$ (polymeric slab), 0.45 (cylinder), and 0.43 (sphere) indicate Fickian diffusion (diffusion controlled). Values of $n=1$ (slab), 0.89 (cylinder), and 0.85 (sphere) suggest case
II transport (swelling controlled, purely relaxation controlled drug delivery). Occasionally the value of $n>1$ (slab), $>0.89$ (cylinder), and $>0.85$ (sphere) has been observed, which has been regarded as the super case II kinetics. Intermediate $n$ values indicate an anomalous behavior, that is, nonFickian kinetics corresponding to couple diffusion/polymer relaxation. In diffusion controlled systems, Fickian diffusion dominates the drug release process, whereas, in swelling controlled delivery systems, the rate of drug release depends on the swelling rate of polymer network [14, 15].

From the $n$ values, formulations J2-J7 followed Fickian diffusion; that is, drug-release is purely controlled by drugdiffusion through the gel layer. Fickian diffusional release occurs by molecular diffusion of the drug due to a chemical potential gradient, whereas formulations $\mathrm{J} 1$ and $\mathrm{J} 9$ followed anomalous non-Fickian diffusion corresponding to coupling of diffusion/polymer relaxation. This type of drug release has been used to describe diffusion of hydrophilic drug from swollen matrix. Formulation J8 followed super case II transport. This kind of release would describe a transport in which the diffusion depends on both the concentration and time and in which the rate of dissolution medium uptake into the polymer matrix is largely determined by the rate of swelling and relaxation of the polymer chains.

\section{Conclusions}

In this study, feasibility of low molecular weight Chitosan in combination with MSA/CMCNa/TSG as polymeric carrier for hydrodynamically balanced capsule formulations was studied using Propranolol Hydrochloride as model drug. All the combination of $\mathrm{LMCH}$ with $\mathrm{MSA} / \mathrm{CMCNa}$ (J1J6) in the ratio of $4: 1$ and $4: 1.5$ (LMCH:MSA/CMCNa) showed good buoyancy which last up to $6 \mathrm{~h}$ in $0.1 \mathrm{M} \mathrm{HCl}$. Reversing the polymeric ratio to $1: 4$ (LMCH:MSA, J8) or completely removing the $\mathrm{LMCH}$ resulted in polymer matrices which remained buoyant for only $4 \mathrm{~h}$ in $0.1 \mathrm{M} \mathrm{HCl}$. The drug release retardation from the formulations (J2-J7) was attributed to the in situ PEC formation between LMCH and MSA/CMCNa. Incorporation of MCCP (rapid hydration) and TSG (Plug formation) was found to be innovative. Considering the experimental data, it may be concluded that 
LMCH in combination with MSA/CMCNa may constitute a potential polymeric carrier for the development of sustained release stomach specific sustained delivery of hydrophilic drugs with absorption window in the upper GIT.

\section{Conflict of Interests}

The authors declare that there is no conflict of interests regarding the publication of this paper.

\section{Acknowledgments}

The authors thank Dr. R. M. Dubey, Vice Chancellor of the IFTM University; Dr. S. D. Sharma, School of Sciences, IFTM University; and Dr. Reddy's Laboratories, India, for generosity.

\section{References}

[1] H. Mohammad, "Gastroretentive drug delivery system comprising an extruded hydratable polymer," EP 1513508 B1, EuroCeltique S.A., 2014.

[2] M. Tanaka, K. Sato, E. Kitakami, S. Kobayashi, T. Hoshiba, and K. Fukushima, "Design of biocompatible and biodegradable polymers based on intermediate water concept," Polymer Journal, vol. 47, no. 2, pp. 114-121, 2015.

[3] D. Hunkeler, K. Eichler, and J. Arnold, "Gastrotentive drug delivery system for controlled drug release in the stomach and into the upper intestines," US $20130315991 \mathrm{Al}$, Aqua+Tech Specialties, La Plaine, Switzerland, 2013.

[4] V. Kumar, S. Ahmad, and B. R. Singh, "A gastroretentive dosage system and process of preparation thereof," Patent US20140271846, Ranbaxy Laboratories, 2013.

[5] B. Bret and J. Louie-Helm, "Gastric retentive oral dosage form with restricted drug release in the lower gastrointestinal tract," US20110301129 A1, 2011.

[6] M. Friedman and D. Kirmayer, "Novel gastroretentive delivery system," US 20110268666 A1, Yissum Research Development Company of the Research University of Jerusalem, 2011.

[7] J. Ogorka and J. N. Kavimandan, "Extended release gastroretentive oral drug delivery system for valsartan," EP 2061438 A1, Novartis AG, 2009.

[8] N. Bhattarai, J. Gunn, and M. Zhang, "Chitosan-based hydrogels for controlled, localized drug delivery," Advanced Drug Delivery Reviews, vol. 62, no. 1, pp. 83-99, 2010.

[9] E. R. Gariépy and J. C. Leroux, "Chitosan: a natural polycation with multiple applications," in Polysaccharides for Drug Delivery and Pharmaceutical Applications, R. H. Marchessault, F. Ravenelle, and X. X. Zhu, Eds., vol. 934 of ACS Symposium Series, pp. 243-259, ACS, 2006.

[10] S. Y. Park, K. S. Marsh, and J. W. Rhim, "Characteristics of different molecular weight chitosan films affected by the type of organic solvents," Journal of Food Science, vol. 67, no. 1, pp. 194-197, 2002.

[11] J. Ali, S. Arora, A. Ahuja et al., "Formulation and development of hydrodynamically balanced system for metformin: in vitro and in vivo evaluation," European Journal of Pharmaceutics and Biopharmaceutics, vol. 67, no. 1, pp. 196-201, 2007.

[12] A. Verma, A. K. Bansal, A. Ghosh, and J. K. Pandit, "Low molecular mass chitosan as carrier for a hydrodynamically balanced system for sustained delivery of ciprofloxacin hydrochloride," Acta Pharmaceutica, vol. 62, no. 2, pp. 237-250, 2012.

[13] US Pharmacopeial Convention, United States Pharmacopoeia 27/National Formulary 22, US Pharmacopeial Convention, Rockville, Md, USA, 2000.

[14] C. Paulo, M. Jose, and L. Sousa, "Modeling and comparison of dissolution profiles," European Journal of Pharmaceutical Sciences, vol. 13, pp. 123-133, 2001.

[15] T. Higuchi, "Rate of release of medicaments from ointment bases containing drugs in suspension," Journal of Pharmaceutical Sciences, vol. 50, pp. 874-875, 1961.

[16] E. L. McConnell, H. M. Fadda, and A. W. Basit, "Gut instincts: explorations in intestinal physiology and drug delivery," International Journal of Pharmaceutics, vol. 364, no. 2, pp. 213-226, 2008.

[17] P. R. Sheth and J. Tossounian, "The hydrodynamically balanced system: a novel drug delivery system for oral use," Drug Development and Industrial Pharmacy, vol. 10, no. 2, pp. 313$339,1984$.

[18] H. Omidian and K. Park, "Swelling agents and devices in oral drug delivery," Journal of Drug Delivery Science and Technology, vol. 18, no. 2, pp. 83-93, 2008.

[19] R. H. G. Brinkhuis and A. J. Schouten, "Thin-film behavior of poly(methyl methacrylates). 4. Stereocomplexation of isotactic and syndiotactic poly(methyl methacrylate) at the air-water interface," Macromolecules, vol. 25, no. 10, pp. 2725-2731, 1992.

[20] K. S. De, N. R. Aluru, B. Johnson, W. C. Crone, D. J. Beebe, and J. Moore, "Equilibrium swelling and kinetics of $\mathrm{pH}$-responsive hydrogels: models, experiments, and simulations," Journal of Microelectromechanical Systems, vol. 11, no. 5, pp. 544-555, 2002.

[21] S. Marco, A. Adonella, and M. Elsa, "Use of a polysaccharide polymer from the seeds of the tamarind tree in protecting against tobacco-associated damages," EP 2741754 Al, Zambon S.p.A., 2014. 

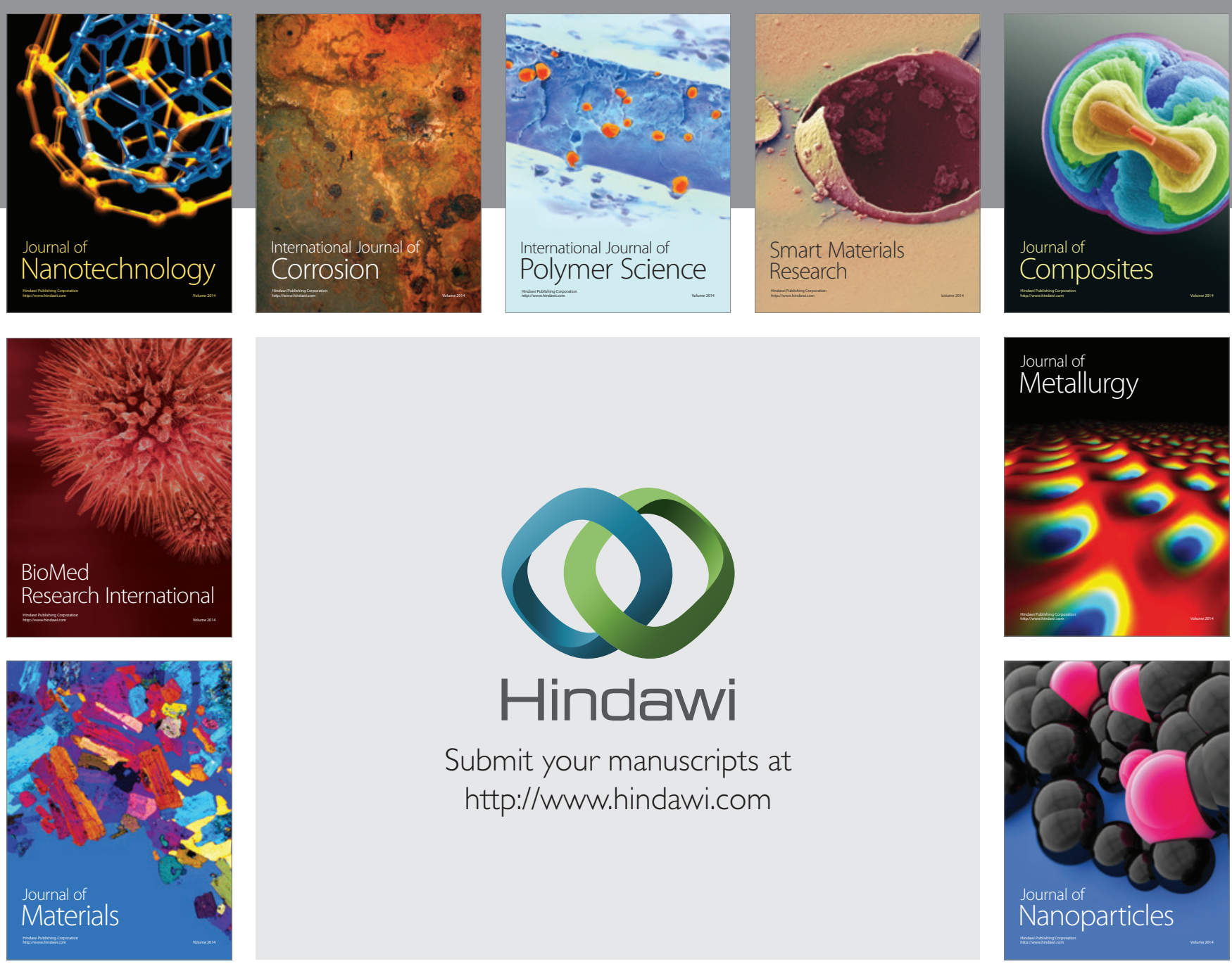

Submit your manuscripts at http://www.hindawi.com
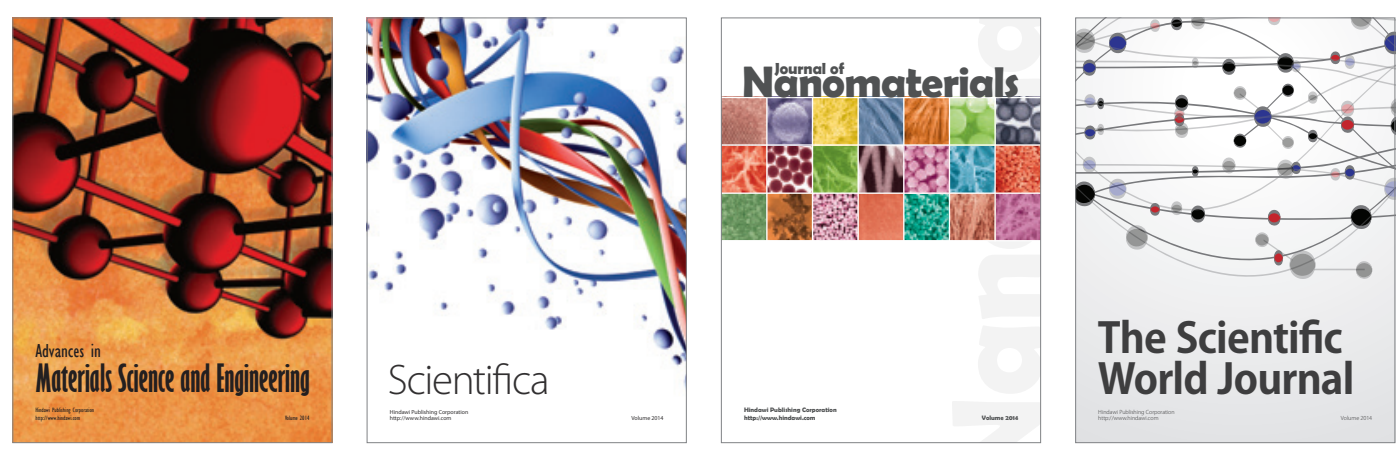

\section{The Scientific World Journal}
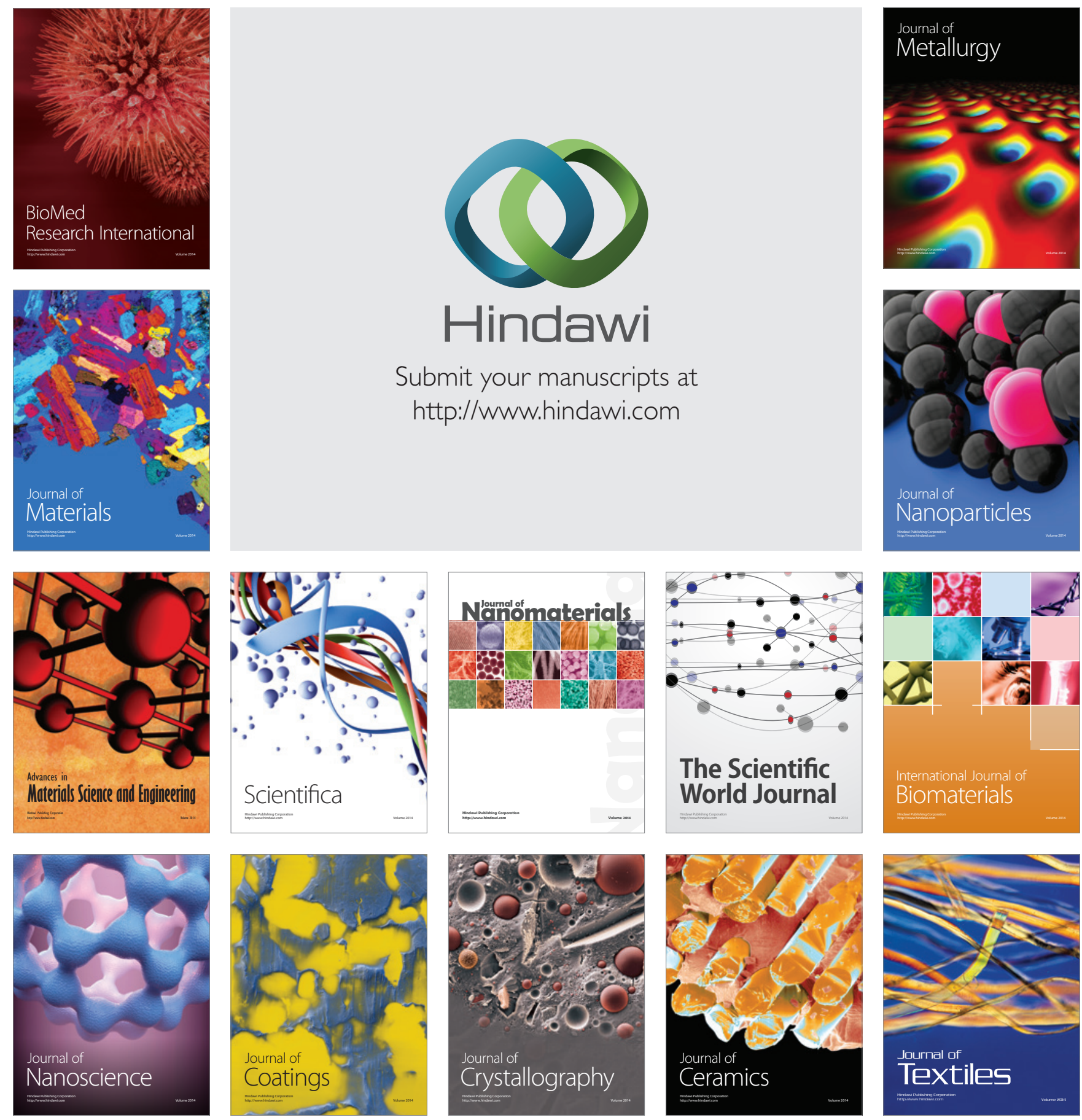